\title{
Pentoxifylline decreases post-operative intra-abdominal adhesion formation in an animal model
}

\author{
Ya-Lin Yang ${ }^{1}$, Meng-Tse Gabriel Lee ${ }^{1}$, Chien-Chang Lee ${ }^{1}{ }^{\text {, Pei-I Su }}{ }^{1}$, Chien-Yu Chi ${ }^{1}$, Cheng-Heng Liu ${ }^{1}$, \\ Meng-Che Wu ${ }^{1}$, Zui-Shen Yen ${ }^{1}$, Shyr-Chyr Chen ${ }^{\text {Corresp. } 1}$ \\ ${ }^{1}$ Department of Emergency Medicine, National Taiwan University Hospital and College of Medicine, Taipei, Taiwan \\ Corresponding Author: Shyr-Chyr Chen \\ Email address: scchen@ntu.edu.tw
}

Background Intra-abdominal adhesions develop after nearly every abdominal surgery, commonly causing female infertility, chronic pelvic pain, and small bowel obstruction. Pentoxifylline (PTX) is a methylxanthine compound with immunomodulatory and antifibrotic properties. The aim of this study was to investigate whether PTX can reduce post-operative intra-abdominal adhesion formation via collagen deposition, tissue plasminogen activator (tPA) level, inflammation, angiogenesis, and fibrosis.

Methods Seventy male BALB/c mice were randomized into one of three groups: (1) sham group without peritoneal adhesion model; (2) peritoneal adhesion model (PA group); (3) peritoneal adhesion model with PTX (100 mg/kg/day i.p.) administration was started on preoperative day 2 and continued daily (PA+PTX group). On postoperative day 3 and day 7, adhesions were assessed using the Lauder scoring system. Parietal peritoneum was obtained for histological evaluation with hematoxylin and eosin (HE) and picrosirius red staining. Fibrinolysis was analyzed by tPA protein levels in the peritoneum by ELISA. Immunohistological analysis was also conducted using markers for angiogenesis $\left(\mathrm{ki}^{+} 7^{+} / \mathrm{CD} 31^{+}\right)$, inflammation $\left(\mathrm{F} 4 / 80^{+}\right.$) and fibrosis $\left(\mathrm{FSP}-1^{+}\right.$and $\left.\alpha-\mathrm{SMA}^{+}\right)$. All the comparisons were made by comparing the PA group with the PTX treated PA group, and $p<0.05$ was considered statistically significant.

Results Intra-abdominal adhesions were markedly reduced by PTX treatment. Compared with the PA group, PTX treatment had lower adhesion scores than the PA group on both day 3 and day $7(p<0.05)$. Histological evaluations found that PTX treatment reduced collagen deposition and adhesion thickening. ELISA analysis showed that PTX treatment significantly increased the level of tPA in the peritoneum. In addition, in the immunohistological analysis, PTX treatment was found to significantly decreased the number of $\mathrm{ki} 67^{+} / \mathrm{CD} 31^{+}$cells at the site of adhesion. Finally, we also observed that in the PTX treated group, there was a reduction in the expression of $\mathrm{F} 4 / 80^{+}, \mathrm{FSP}-1^{+}$, and $\alpha-\mathrm{SMA}^{+}$cells at the site of adhesion.

Conclusion PTX may decrease intra-abdominal adhesion formation via increasing peritoneal fibrinolytic activity, suppressing angiogenesis, decreasing collagen synthesis, and reducing peritoneal fibrosis. Our findings suggest that PTX can be used to decrease post-operative intra-abdominal adhesion formation. 
1 Pentoxifylline decreases post-operative intra-abdominal adhesion formation in an animal 2 model

3

4 Ya-Lin Yang ${ }^{1 \ddagger}$, Meng-Tse Gabriel Lee ${ }^{1 \ddagger}$, Chien-Chang Lee ${ }^{1}$, Pei-I Su${ }^{1}$, Chien-Yu Chi ${ }^{1}$, Cheng5 Heng Liu ${ }^{1}$, Meng-Che Wu${ }^{1}$, Zui-Shen Yen ${ }^{1}$, Shyr-Chyr Chen ${ }^{1 *}$

6 1. Department of Emergency Medicine, National Taiwan University Hospital and College of 7 Medicine, National Taiwan University, Taipei, Taiwan.

8

$9 \$$ The two authors contribute equally to this work

10 * Correspondence and address reprint request to: Dr. Shyr-Chyr Chen

11 Department of Emergency Medicine, National Taiwan University Hospital and College of 12 Medicine, National Taiwan University, Taipei, Taiwan.

$13 *$ scchen@ntu.edu.tw

14 
16

17 18

\section{Background}

\section{Abstract}

Intra-abdominal adhesions develop after nearly every abdominal surgery, commonly causing female infertility, chronic pelvic pain, and small bowel obstruction. Pentoxifylline (PTX) is a methylxanthine compound with immunomodulatory and antifibrotic properties. The aim of this study was to investigate whether PTX can reduce post-operative intra-abdominal adhesion formation via collagen deposition, tissue plasminogen activator (tPA) level, inflammation, angiogenesis, and fibrosis.

\section{Methods}

Seventy male BALB/c mice were randomized into one of three groups: (1) sham group without peritoneal adhesion model; (2) peritoneal adhesion model (PA group); (3) peritoneal adhesion model with PTX (100 mg/kg/day i.p.) administration was started on preoperative day 2 and continued daily (PA+PTX group). On postoperative day 3 and day 7, adhesions were assessed using the Lauder scoring system. Parietal peritoneum was obtained for histological evaluation with hematoxylin and eosin (HE) and picrosirius red staining. Fibrinolysis was analyzed by tPA protein levels in the peritoneum by ELISA. Immunohistological analysis was also conducted using markers for angiogenesis $\left(\mathrm{ki}^{+} 7^{+} / \mathrm{CD} 31^{+}\right)$, inflammation $\left(\mathrm{F} 4 / 80^{+}\right)$and fibrosis $\left(\mathrm{FSP}-1^{+}\right.$and $\alpha$-SMA ${ }^{+}$). All the comparisons were made by comparing the PA group with the PTX treated PA group, and $p<0.05$ was considered statistically significant.

\section{Results}

Intra-abdominal adhesions were markedly reduced by PTX treatment. Compared with the PA group, PTX treatment had lower adhesion scores than the PA group on both day 3 and day $7(p<0.05)$. Histological evaluations found that PTX treatment reduced collagen deposition and adhesion thickening. ELISA analysis showed that PTX treatment significantly increased the level of tPA in the peritoneum. In addition, in the immunohistological analysis, PTX treatment was found to significantly decreased the number of $\mathrm{ki} 67^{+} / \mathrm{CD} 31^{+}$cells at the site of adhesion. Finally, we also observed that in the PTX treated group, there was a reduction in the expression of $\mathrm{F} 4 / 80^{+}, \mathrm{FSP}-1^{+}$, and $\alpha-\mathrm{SMA}^{+}$cells at the site of adhesion.

\section{Conclusion}

PTX may decrease intra-abdominal adhesion formation via increasing peritoneal fibrinolytic activity, suppressing angiogenesis, decreasing collagen synthesis, and reducing peritoneal fibrosis. Our findings suggest that PTX can be used to decrease post-operative intra-abdominal adhesion formation. 


\section{Introduction}

52 Post-operative intra-abdominal adhesion after laparotomy is a source of considerable morbidity.

53 It is estimated that more than $90 \%$ of patients develop primary intra-abdominal adhesion after 54 laparotomy. Post-operative adhesions affect the quality of life in millions of people worldwide, 55 causing many different types of complications, including chronic pelvic or abdominal pain, small 56 bowel obstructions (SBO), and even infertility (Arung et al. 2011; Liakakos et al. 2001). SBO is 57 the most common complication of adhesion and is observed in up to $70 \%$ of patients undergoing 58 laparotomy (Ellis 1997; Menzies \& Ellis 1990; ten Broek et al. 2013). Although less commonly 59 observed, up to $20 \%$ of female infertility has been associated with post-operative adhesions 60 (Luijendijk et al. 1996).

61 The pathogenesis of post-operative intra-abdominal adhesion is a complex process that involves 62 inflammation, collagen related clot formation, angiogenesis, fibrinolysis, and tissue repairs 63 which include epithelial-mesenchymal transition (EMT)/endothelial-mesenchymal transition 64 (EndMT) or mesothelial -mesenchymal transition (MMT) (diZerega 1997; Hellebrekers \& 65 66 Kooistra 2011; Homdahl \& Ivarsson 1999). The key area in adhesion formation is the surface lining of the peritoneum. Injury of the peritoneum leads to activation of coagulation cascade and an inflammatory response consisting of hyperemia, fluid exudation, recruitment of floating mesothelial cells, and release of white blood cells and platelets into the peritoneal cavity(diZerega 1997; diZeregal \& Campeau 2001). Normal fibrinolytic activity usually prevents fibrinous attachments for 3-4 day, and mesothelial repair occurs in 5-6 day after surgery (diZeregal \& Campeau 2001). Therefore, previous studies focused on the cellular events 3-6 day after the peritoneal injury.

Pentoxifylline (PTX), a non-specific phosphodiesterase inhibitors, has been used to improve the walking ability in patients with intermittent claudication (Ernst 1994; Hood et al. 1996; Rossner \& Muller 1987). Previous animal studies have also demonstrated that PTX can reduce postoperative adhesion, but the biological mechanisms that were responsible have not been fully clarified (Durmus et al. 2011; Hung et al. 2008; Jafari-Sabet et al. 2015; Tarhan et al. 2006). Five separate mechanisms on how PTX can alter the essential components in adhesion formation have been proposed: (1) reduction of inflammation (Durmus et al. 2011; Pollice et al. 2001); (2) reduction of collagen synthesis (Chen et al. 1999); (3) reduction of angiogenesis (Amirkhosravi et al. 1998) (4) increased fibrinolysis by up-regulation of tissue plasminogen activator (tPA) expression (Tarhan et al. 2006); (5) reduced fibrosis (Durmus et al. 2011; Wen et al. 2017). However, it is unclear if PTX can decrease post-operative intra-abdominal adhesion formation by simultaneously altering all of the five proposed mechanisms. Therefore, we aimed to 
85 investigate the effects of PTX on peritoneum collagen expression, peritoneal tPA expression, 86 peritoneum angiogenesis, inflammation, and peritoneal fibrosis.

87

88

89

90

91

92

93

94

95

96

97

98

99

100

101

102

103

104

105

106

107

108

109

110

111

112

113

114

115

116

\section{Methods}

\section{Animals}

Male BALB/c mice weighing 25-30 g (Charles River Laboratories, BioLasco, Taiwan) were maintained in a temperature and light-controlled room (12-hour light/dark cycle) and allowed free access to water and food. The experimental protocols were approved by the Institutional Animal Care and Use Committee (IACUCs) of the National Taiwan University Hospital (approval ID: 20120285). All the protocols are in adherence to the guidelines established in the Guide for the Care and Use of Laboratory Animals of the National Health Research Institutes.

\section{Experimental design}

$70 \mathrm{BALB} / \mathrm{c}$ mice were randomly divided into three different groups: (1) sham group without peritoneal adhesion model (sham group, $\mathrm{n}=8$ ); (2) peritoneal adhesion model (PA group, $\mathrm{n}=$ 31); (3) peritoneal adhesion model with PTX (Trental ) (100 mg/kg/day i.p., once daily) administration was started on preoperative day 2 and continued daily (PA+PTX group, $\mathrm{n}=31$ ). We used a slightly modified standard adhesion model (Oncel et al. 2001). The details for peritoneal adhesion model had been reported before (Lee et al. 2016).

Briefly, mice were anesthetized using $2 \%$ isoflurane in oxygen. The abdomen was then shaved and disinfected with povidone iodine. A $4 \mathrm{~cm}$ median laparotomy was performed to gain access to the abdominal cavity. In peritoneal adhesion model (PA and PA+PTX group), were pooled and randomly underwent surgery. The cecum was gently removed and abraded with 20 vertically reciprocal movements of dry gauze. After, the right abdominal sidewall be more aggressive with the abraded than cecum and until punctate bleeding was seen. The injury sites were cleaned with physiological salt solution and covered the gauze, making sure that was no active bleeding. The cecum was then placed back into the abdominal cavity and surgical wound was sutured. For the sham group, only open laparotomy and closure was conducted and there is no abrading of cecum and abdominal wall.

The PTX group received $100 \mathrm{mg} / \mathrm{kg}$ of PTX from left abdominal cavity, whereas the other two group (group sham and PA) received $0.125 \mathrm{ml}$ of physiological saline solution. For preventing postoperative pain, buprenorphine $(0.05 \mathrm{mg} / \mathrm{kg}$ s.c., twice daily) was administered during the two postoperative days. Mice were placed under a warming lamp and observed until they recovered 
117 fully from anesthesia. Mice were monitored daily for signs of wound infection and general health

118 condition periodically until 3 or 7 day after surgery.

\section{Adhesion score}

120 Mice were euthanized on postoperative day 3 and day 7. The abdominal cavity was opened via a

121 U-shaped incision. The adhesion score was evaluated was performed by an observer blinded

122 treatment, using the Lauder scoring system (Lauder et al. 2011). The adhesion were graded in a

123 blinded fashion using the classification system described (Table 1).

\section{Histology staining}

125 Tissue samples from the parietal peritoneum, liver, and mesentery were collected after 126 euthanasia. For histological staining, tissue were fixed in 10\% neutral-buffered formalin (NBF), 127 paraffin embedded, thinly sectioned. Tissue sections of $4-5 \mu \mathrm{m}$ thickness were prepared for 128 staining. After deparaffinization and rehydration, sections were counterstained in Gill's 129 hematoxylin (Sigma, St Louis, MO) and for $5 \mathrm{~min}$, cleared in $0.1 \%$ acid alcohol for $30 \mathrm{sec}$, and 130 rinsed in tap water, then stained in eosin (Sigma) for $2 \mathrm{~min}$, cleared in 95\% alcohol, and rinsed $13170 \%$ alcohol to remove the staining solution, dehydrated, and mounted, for histologic assessment.

\section{Picrosirius red staining}

134 Picrosirius red staining was used to compare collagen and fibrosis in tissue between different groups. Peritoneal sections $(4-5 \mu \mathrm{m})$ were deparaffinized, rehydrated and then subjection to counterstaining in Gill's hematoxylin (Sigma) for $5 \mathrm{~min}$, cleared in $0.1 \%$ acid alcohol $30 \mathrm{sec}$, and rinsed in running tap water. Then stained in picrosirius red stain kit (polysciences, Warrington, PA). Subsequently, sections were dehydrated and mounted for assessment.

\section{Tissue plasminogen activator}

140 Peritoneal tissue were prepared by grinding on ice in radioimmunoprecipitation assay buffer 141 (RIPA buffer) with protease inhibitor cocktail (Sigma). After the samples were centrifuged at $14212,000 \mathrm{~g}$ for $15 \mathrm{~min}$ at $4^{\circ} \mathrm{C}$, supernatants were aspirated and placed in new tubes. Samples were 143 analyzed for total antigen concentration of tPA, using commercially available ELISA kits from 144 Molecular Innovations (Molecular Innovations, Novi, MI). Total protein content was determined 145 by Bradford assay (Sigma).

\section{Immunohistochemistry}


147 Formalin-fixed and paraffin-embedded peritoneal tissue was sectioned at 4-5 $\mu \mathrm{m}$ and then 148 subjected to double immunostaining. Briefly, sections were deparaffinized, rehydrated and 149 endogenous peroxidase activity was quenched by $3 \%$ hydrogen peroxide $\left(\mathrm{H}_{2} \mathrm{O}_{2}\right)$ for 10 min. 150 Sections were subjected to antigen retrieval was performed in $\mathrm{pH} 6.0$ citrate buffer using a 151 microwave oven for 15 min. Blocking of non-specific binding was done by incubation with $2.5 \%$ 152 horse serum at room temperature for $30 \mathrm{~min}$. Sections were incubated with primary antibodies, 153 rabbite anti-ki67 (1:200, Abcam, Cambridge, MA), rat anti-F4/80 (1:200, Abcam) or rabbit anti154 FSP-1 (1:200, Abcam) overnight at $4^{\circ} \mathrm{C}$. After washing with Tris-buffered saline (TBS, pH7.4), 155 sections were incubated using the ImmPRESS AP anti-rabbit polymer reagent (Vector 156 Laboratories, Burlingame, CA) for $30 \mathrm{~min}$ at room temperature. Positive signal resulted in blue 157 nuclear staining with the VECTOR Blue kit (Vector Laboratories). After washing and blocking 158 again, the sections were incubated with goat anti-CD31 (1:500, R\&D System, Minneapolis, MN) 159 for 1 hour at room temperature. After washing, ImmPRESS HRP anti-goat polymer reagent 160 (Vector Laboratories) was used for $30 \mathrm{~min}$ at room temperature. Positive reactions for 161 endothelial cells resulted in brown red staining with the NovaRed substrate kit (Vector 162 Laboratories). Sections were examined by light microscopy (Nikon Instruments, Nikon 163 Corporation, Tokyo, Japan).

\section{Immunofluorescence staining}

165 Peritoneal tissue sections $(4-5 \mu \mathrm{m})$ were performed for double immunofluorescence staining. 166 Briefly, sections were deparaffinized, rehydrated and were treated with $0.3 \% \mathrm{H}_{2} \mathrm{O}_{2}$ for 10 min to 167 block endogenous peroxide activity and boiled in $\mathrm{pH} 6.0$ citrate buffer using a microwave oven

168 for $15 \mathrm{~min}$. Sections were subsequently incubated with 5\% Donkey serum for $20 \mathrm{~min}$ at room 169 temperature. Sections were incubated with rabbit anti-cytokeratin 18 (CK18) (1:200, Enogene, 170 New York, NY) overnight at $4^{\circ} \mathrm{C}$, washed in phosphate-buffered saline (PBS, pH7.4) and 171 incubated using donkey anti-rabbit DyLight 488 antibody (Thermo Scientific, Rockford, IL) and 172 Cy3-conjugated mouse anti- $\alpha$-smooth muscle actin ( $\alpha$-SMA) (Sigma) and then mounted and 173 subjected to fluorescence microscopy (Leica DMRA, Leica Microsystems, Wetzlar, Germany). 174 Images were recorded at x100, x200 and x400 magnification of light microscopy, which were 175 then digitalized and analyzed using Image-Pro Plus 6.0 software (Media Cybernetics, Rockville, $176 \mathrm{MD})$.

\section{Statistical Analysis}

178 Normal distributed continuous data were expressed as mean \pm standard error (SE). For non179 parametric data, results were expressed as median \pm interquartile range (IQR). The difference between continuous variables were evaluated using one-way ANOVA when data distribution 
181 was normal and a Mann-Whitney test was used for non-normal distributed continuous data. A $p$ 182 value of less than 0.05 was considered statistically significant. The statistical analyses were 183 performed with GraphPad Prism (version 6.0, GraphPad Software, Inc., La Jolla).

184

185

186

187

188

189

190

191

192

193

194

195

196

197 198

199

200

201

202

203

204

205

206

207

208

209

210

\section{Results}

\section{Deaths of animal}

Surgical procedures were successfully completed on 69 animals, except for one mice in the sham group, which died due to anesthesia-related complication before the commencement of surgery. One mice in the PA+PTX group died during recovery from anesthesia. Four mice died within 48 hour of surgery and were excluded from the study (PA and PA+PTX group, $n=2$ /group). Two mice in the PA group were excluded due to severe distress, according to three criteria in Health Evaluation of Experimental Laboratory Mice: very rough hair coat, hunched, and not eating or drinking. No mice in the PA+PTX treated group incurred any life-threatening side effects or deaths at 48 hours after surgery, which lead to exclusion from the study. Therefore, a total of 8 animals were excluded from the study.

Mice were euthanatized for the planned experiments on postoperative day 3 (PA group, $\mathrm{n}=14$; PA+PTX group, $\mathrm{n}=13$ ) and day 7 (sham group, $\mathrm{n}=7$; PA group, $\mathrm{n}=13$; PA+PTX group, $\mathrm{n}=$ $15)$.

\section{Pentoxifylline Treatment Reduces Adhesion score}

Total adhesion scores data was examined and plotted for post-operative day 3, and 7 (Figure 1). We observed that the sham group, which had not undergone the adhesion model, had significantly lower adhesion score than the animals that had undergone adhesion model, as expected. PA+PTX group (median, 1.00; IQR, 0.50-2.00) had significantly lower adhesion score than the PA group (median, 2.00; IQR, 2.00-3.00) on day $3(p<0.05)$. On day 7, mice treated with PTX (median, 3.00; IQR, 0.00-3.00) still had lower adhesion score than the PA group (median, 3.00; IQR, 3.00-4.50) $(p<0.05)$.

\section{Pentoxifylline Treatment Inhibits Collagen Deposition}

We used the HE staining to compare changes in peritoneal structure (Figure 2A). In general, there were increased thickness of submesothelial layer on day 3, and the adhesion score also increased. We observed that the sham group, had the thin submesothelial layer as demonstrated 
211 in (Figure 2A). The severe adhesion and thick submesothelial layer was observed, as well as 212 increased cellularity in the PA group. In contrast, PA+PTX group had less peritoneal 213 submesothelial thickness and adhesion severity as compared with the PA group.

214 We further used picrosirius red staining to assess the quality of collagen fiber in peritoneal 215 adhesion (Figure 2B). Compared with PA group, PA+PTX group had less collagen deposition 216 and the thickness of the abdominal adhesions. Our data suggested that PTX could decrease 217 collagen deposition during adhesion formation.

\section{Pentoxifylline Treatment Increased tPA level}

219 The tPA protein levels in the peritoneum of mice were perceived to be measured and plotted on 220 post-operative day 3 and 7 (Figure 3). We observed that the sham group of mice had the lowest 221 tPA protein level throughout the study period $(p<0.001)$. Those mice were treated with PTX had 222 higher tPA protein level than those untreated mice (sham and PA group). There was significant 223 difference between PA+PTX group and PA group on day 7 (0.365 \pm 0.024 vs. $0.193 \pm 0.03, p<$ $2240.001)$.

225

226

\section{Pentoxifylline Treatment Reduced Angiogenesis}

227 We performed immunohistochemical staining assay to analyze the status of angiogenesis during 228 peritoneal repair. Proliferating endothelial cells were identified as those cells with cytoplasmic 229 CD31 staining and nuclear Ki67 staining (Figure 4A, arrows). Cells that staining positive for 230 CD31 but without nuclear staining for Ki67 were scored as nonproliferating endothelial cells. 231 We examined the effect of endothelial cell proliferation in peritoneum was quantified by 232 measuring the number of $\mathrm{ki}^{+} 7^{+} \mathrm{CD} 31^{+}$cell at the site of adhesion (Figure 4B). Our results 233 showed that PA group had significantly higher proliferating endothelial cells compared to both 234 sham and PA+PTX group on day 3 and day 7(all $p<0.001)$. We also observed that number of $235 \mathrm{ki}^{+} 7^{+} \mathrm{CD} 31^{+}$proliferating endothelial cell count decrease substantially over time $(p<0.001)$ in 236 the PA group. Otherwise, we examined the angiogenic effect in peritoneum by measuring the 237 area of $\mathrm{CD} 1^{+}$microvessel at the site of adhesion (Figure $4 \mathrm{C}$ ). We also observed that the PA 238 group, which had vessel coverage (percentage of area covered by CD $31^{+}$per field) of $1.63 \%$ at day 3, and 3.63\% at day 7, respectively. On post-operative day 7, PA group demonstrated an 240 increase in CD31 expression compared to the day 3 and indicated that blood vessel formation 241 was significantly more prominent in the PA group compared to PA+PTX groups $(p<0.001)$. Thus, 242 PTX can significantly suppress angiogenesis during peritoneal repair. 


\section{Pentoxifylline Treatment Reduced Inflammation}

244 Inflammation, an important component both in normal and pathological healing, is a protective

245

246

247

248

249

250

251

252

253

254

255

256

257

258

259

260

261

262

263

264

265

266

267

268

269

270

271

272

273

274

275

276

277

response of the tissue injury, designed for removal of the causative agent and restoration of tissue structure and function. We performed immunohistochemical staining assay to analyze the infiltration of macrophage during peritoneal repair. F4/80 is a macrophage-specific marker in the mice. As shown in figure $5 \mathrm{~A}$, a large number of $\mathrm{F} 4 / 80^{+}$-expressed cells were observed in the PA group. Quantification analysis of IHC image revealed significantly increased expression of $\mathrm{F} 4 / 80^{+}$cells on day $3(0.80 \% \pm 0.10 \%)$, and highest expression on day $7(2.56 \% \pm 0.22 \%)$ in the PA group as compared to the sham group ( $p<0.01$ for both day 3 and day 7$)$ or PA+PTX group $(1.37 \% \pm 0.28 \%, p<0.01$ on day 7 , Figure $5 \mathrm{~B})$. PA+PTX group had significantly lower expression of $\mathrm{F} 4 / 80^{+}$as compared with PA group.

\section{Pentoxifylline Treatment Reduced the Expression of Fibrosis Marker FSP-1}

FSP-1, also known as fibroblast-specific protein 1 (FSP1), belongs to the S100 superfamily of cytoplasmic calcium-binding proteins and can be expressed by cell of mesenchymal origin or fibroblastic phenotype. This protein is reported to be specific for fibroblasts and to play a causal role in EMT. As shown in figure $6 \mathrm{~A}$ and $6 \mathrm{~B}$, a large number of FSP- ${ }^{+}$-expressed cells were observed in the PA group. Quantification analysis of IHC image revealed significantly increased expression of $\mathrm{FSP}_{-} 1^{+}$on day $3(5.37 \% \pm 1.03 \%)$, and highest expression on day $7(11.26 \% \pm$ $1.66 \%$ ) in the PA group compared to the PA+PTX group ( $p<0.05$ for both day 3 and day 7 , Figure 6C). Consistently, we found the mice were treated with PTX had significantly reduced expression of $\mathrm{FSP}^{+}$as compared with PA group.

\section{Pentoxifylline Treatment Reduced the Expression of Fibrosis Marker $\alpha-S M A$}

As a response to injury, mesothelium is undergoing change to mesothelial-to-mesenchymal transition (MMT). Thus, we further performed double immunofluorescence staining for CK18 and $\alpha$-SMA for peritoneal injury (Figure 6, A and B). Many studies have demonstrated that mesenchymal cell markers, including $\alpha$-SMA, is proposed as indicator of EMT (Margetts et al. 2005). Cytokeratin (CK) are structural marker proteins specific for epithelial cell, and CK18 is highly expressed in mesothelial cells. $\alpha$-SMA has become the most reliable marker of myofibroblasts. Figure 7A are consistent with previous studies, in the PA group observed a few $\mathrm{CK} 18^{+} \alpha-\mathrm{SMA}^{+}$double-positive cells appear first in the mesothelial monolayer and later in the reorganized submesothelial matrix. We examined the extent of accumulation of myofibroblasts in peritoneum was quantified by assessing the percentage $\alpha-\mathrm{SMA}^{+}$cells (Figure 7C). Our result showed that PA group had significantly increased $\alpha-\mathrm{SMA}^{+}$expression at day $3(3.48 \% \pm 1.28 \%)$, and highest expression at day $7(13.71 \% \pm 1.40 \%)$ compared with the PA+PTX group $(p<0.05$ for day 3 and $p<0.01$ for day 7 , respectively). PTX significantly attenuated thickening of fibrotic 
278 peritoneum, and accumulation of $\alpha \mathrm{SMA}^{+}$myofibroblasts in peritoneum after injury. 


\section{Discussion}

280 In this study, we demonstrated that PTX treatment could effectively reduce post-operative intra281 abdominal adhesion formation. PTX could prevent peritoneum adhesion formation via 5 related 282 biological processes: increasing fibrinolysis; reducing inflammation; reducing angiogenesis; 283 reducing collagen deposition; and reducing fibrosis.

284 Post-operative intra-abdominal adhesion formation is considered to be an inevitable result of 285 peritoneum injury after abdominal surgery. Peritoneum injury initiates an inflammatory response, 286 which increase in vascular permeability leading to fibrin release and adhesion formation 287 (diZeregal \& Campeau 2001). Under normal conditions, the majority of fibrin is degraded within 288 a few days by locally released proteases of the fibrinolytic system (Harris et al. 1995; Sulaiman 289 et al. 2002). In a pathological state, fibrinolysis does not occur within 5-7 days of the peritoneal 290 injury, the provisional fibrin matrix persists and more gradually becomes organized as the 291 collagen-secreting myofibroblasts and other repairing cell infiltrate the matrix (Homdahl \& 292 Ivarsson 1999). This process leads to peritoneal adhesion and new blood vessel formation 293 (angiogenesis) (Saltzman et al. 1996).

294 We hypothesized that there are at least four mechanisms that PTX treatment might result in 295 reduce post-operative adhesion. First, PTX has been reported to alter rheological properties of 296 blood such as: decreasing blood viscosity by stimulating fibrinolysis to reduce plasma fibrinogen 297 concentrations, increasing erythrocyte flexibility and platelet deaggregation, and inhibiting 298 neutrophil activity to reduce the tissue damage (McCarty et al. 2016). The alteration in the 299 rheological properties of blood may be the reason why we observed that the tPA level was 300 significantly higher in the PTX treated group than those without PTX treatment group. In fact, 301 we have previously found that mice treated with therapeutic hypothermia, have increased tPA 302 levels and reduced post-operative adhesion (Lee et al. 2016).

303 Second, the anti-inflammation property of PTX has been well established by several previous 304 studies, and has been found to attenuate the cardiopulmonary bypass (CPB)-induced systemic 305 inflammatory response syndrome and postoperative mortality (Barkhordari et al. 2011; Heinze et 306 al. 2007; Otani et al. 2008). PTX has been found to affect inflammation by reducing the plasma 307 levels of pro-inflammatory cytokines such as TNF- $\alpha$, IL-1 and IL-6 (Otani et al. 2008; Pollice et 
308 al. 2001). The reduction of cytokines at the site of injury may explain why we observed a 309 reduction in the infiltration of macrophage in the PTX treated group.

310 Third, PTX also has been reported by previous studies to inhibit endothelial cell proliferation and 311 angiogenesis (Gude et al. 2001; Hasebe et al. 2000). Vlahos et al. reported that PTX might 312 cause suppression of endometriotic lesions by suppressing angiogenesis through VEGF-C and 313 flk-1 expression (Vlahos et al. 2010). Recent evidence also found that PTX inhibits PKC314 dependent activation of NFאB and prevent hypoxia-induced expression of VEGF (Amirkhosravi 315 et al. 1998). Our results on reduction in angiogenesis in PTX treated groups correspond with the 316 above findings.

317 Fourth, PTX was reported by previous studies to down regulate the intracellular signaling of 318 TGF- $\beta$; which can affect collagen synthesis and fibrosis through the cAMP-PKA pathway (Fang 319 et al. 2000; Kucich et al. 2000). Through PKA, PTX has been found to reduce TGF- $\beta$-induced 320 collagen synthesis in vascular smooth muscle cells and human peritoneal mesothelial cells (Chen 321 et al. 1999; Hung et al. 2003). This might explain why we observed lower amount of collagen 322 deposition in PTX treated mice. Moreover, TGF- $\beta 1$ has been reported to be the key initiating 323 factor of fibrosis, and is also known to strongly induce EMT or EndMT (Lamouille et al. 2014; 324 Piera-Velazquez et al. 2011). EMT is defined as a cellular and molecular changes that are usually 325 characterized by loss of cell-cell adhesion, the down-regulation of E-cadherin and other 326 epithelial genes, accompanied by the acquisition of mesenchymal cell morphology, increased 327 contractility and actin stress fibers. This might explain why we observed reduction in markers of 328 fibrosis with PTX treatment.

329 In this study, we found that PTX treatment decreased intra-abdominal adhesion formation by 330 reducing fibrosis, but it is not in our initial objective to confirm whether the reduction in fibrosis 331 might affect general wound healing. The main reason is because several studies have already 332 found that PTX can instead improve general wound healing. Parra-Membrives et al. (2007) 333 showed that PTX improved healing of experimental ischemic colorectal anastomoses by 334 reducing wound and intra-abdominal infections, adhesion formation, and leaks. Comert et al. 335 (2000) showed PTX has positive effect of the obstructive jaundice on healing of intestinal 336 anastomosis healing by suppressing endotoxin-induced TNF- $\alpha$ release from macrophages and 337 monocyte and stabilizing effect on the neutrophils. Therefore, future studies may need to clarify 
338 the time-frame on how PTX treatment can reduce fibrosis and yet improve wound healing, 339 before clinical trial of PTX can be recommend on post-operative patients. In addition, future 340 studies can also clarify the mechanism on how streptokinase interact synergistically with PTX to 341 reduce post-operative adhesion (Jafari-Sabet et al. 2015).

\section{Conclusion}

343 In conclusion, our study showed that PTX may decrease intra-abdominal adhesion formation via 344 increasing peritoneal fibrinolytic activity, reducing inflammation, suppressing angiogenesis, 345 decreasing collagen synthesis, fibroblast producing and peritoneal fibrosis. We believe that 346 future studies should take into the account that PTX can reduce intra-abdominal adhesion 347 formation through multiple pathways.

\section{Acknowledgments}

349 We thank the staff of the Core Labs, the Department of Medical Research, and National Taiwan 350 University Hospital for technical support

351

\section{References}

Amirkhosravi A, Meyer T, Warnes G, Amaya M, Malik Z, Biggerstaff JP, Siddiqui FA, Sherman P, and Francis JL. 1998. Pentoxifylline inhibits hypoxia-induced upregulation of tumor cell tissue factor and vascular endothelial growth factor. Thrombosis and Haemostasis

357

358

359

360

361

362

363

364

365

366

367

368

369 80:598-602.

Arung W, Meurisse M, and Detry O. 2011. Pathophysiology and prevention of postoperative peritoneal adhesions. World Journal of Gastroenterology 17:4545-4553. 10.3748/wjg.v17.i41.4545

Barkhordari K, Karimi A, Shafiee A, Soltaninia H, Khatami MR, Abbasi K, Yousefshahi F, Haghighat B, and Brown V. 2011. Effect of pentoxifylline on preventing acute kidney injury after cardiac surgery by measuring urinary neutrophil gelatinase - associated lipocalin. Journal of Cardiothoracic Surgery 6. Artn 8

10.1186/1749-8090-6-8

Chen YM, Wu KD, Tsai TJ, and Hsieh BS. 1999. Pentoxifylline inhibits PDGF-induced proliferation of and TGF-beta-stimulated collagen synthesis by vascular smooth muscle cells. Journal of Molecular and Cellular Cardiology 31:773-783. DOI 10.1006/jmcc. 1998.0910

Comert M, Taneri F, Tekin E, Ersoy E, Oktemer S, Onuk E, Duzgun E, and Ayoglu F. 2000. The 
370

371

372

373

374

375

376

377

378

379

380

381

382

383

384

385

386

387

388

389

390

391

392

393

394

395

396

397

398

399

400

401

402

403

404

405

effect of pentoxifylline on the healing of intestinal anastomosis in rats with experimental obstructive jaundice. Surgery Today-the Japanese Journal of Surgery 30:896-902.

diZerega GS. 1997. Biochemical events in peritoneal tissue repair. European Journal of Surgery 163:10-16.

diZeregal GS, and Campeau JD. 2001. Peritoneal repair and post-surgical adhesion formation. Hum Reprod Update 7:547-555. DOI 10.1093/humupd/7.6.547

Durmus AS, Yildiz H, Yaman M, and Simsek H. 2011. The effects of heparin and pentoxifylline on prevention of intra-abdominal adhesions in rat uterine horn models: histopathological and biochemical evaluations. Revue Med Vet 162:198-203.

Ellis H. 1997. The clinical significance of adhesions: Focus on intestinal obstruction. European Journal of Surgery 163:5-9.

Ernst E. 1994. Pentoxifylline for Intermittent Claudication a Critical-Review. Angiology 45:339345. Doi 10.1177/000331979404500502

Fang CC, Yen CJ, Chen YM, Shyu RS, Tsai TJ, Lee PH, and Hsieh BS. 2000. Pentoxifylline inhibits human peritoneal mesothelial cell growth and collagen synthesis: effects on TGF-beta. Kidney Int 57:2626-2633. 10.1046/j.1523-1755.2000.00123.x

Gude RP, Binda MM, Boquete AL, and Bonfil RD. 2001. Inhibition of endothelial cell proliferation and tumor-induced angiogenesis by pentoxifylline. Journal of Cancer Research and Clinical Oncology 127:625-630. DOI 10.1007/s004320100262

Harris ES, Morgan RF, and Rodeheaver GT. 1995. Analysis of the Kinetics of Peritoneal Adhesion Formation in the Rat and Evaluation of Potential Antiadhesive Agents. Surgery 117:663-669. Doi 10.1016/S0039-6060(95)80010-7

Hasebe Y, Thomson LR, and Dorey CK. 2000. Pentoxifylline inhibition of vasculogenesis in the neonatal rat retina. Investigative Ophthalmology \& Visual Science 41:2774-2778.

Heinze H, Rosemann C, Weber C, Heinrichs G, Bahlmann L, Misfeld M, Heringlake M, and Eichler W. 2007. A single prophylactic dose of pentoxifylline reduces high dependency unit time in cardiac surgery - a prospective randomized and controlled study. European Journal of Cardio-Thoracic Surgery 32:83-89. 10.1016/j.ejcts.2007.04.01

Hellebrekers BWJ, and Kooistra T. 2011. Pathogenesis of postoperative adhesion formation. British Journal of Surgery 98:1503-1516. 10.1002/bjs. 7657

Homdahl L, and Ivarsson ML. 1999. The role of cytokines, coagulation, and fibrinolysis in peritoneal tissue repair. European Journal of Surgery 165:1012-1019.

Hood SC, Moher D, and Barber CG. 1996. Management of intermittent claudication with pentoxifylline: Meta-analysis of randomized controlled trials. Canadian Medical Association Journal 155:1053-1059.

Hung KY, Huang JW, Chen CT, Lee PH, and Tsai TJ. 2003. Pentoxifylline modulates 
406

407

408

409

410

411

412

413

414

415

416

417

418

419

420

421

422

423

424

425

426

427

428

429

430

431

432

433

434

435

436

437

438

439

440

441

intracellular signalling of TGF-beta in cultured human peritoneal mesothelial cells: implications for prevention of encapsulating peritoneal sclerosis. Nephrol Dial Transplant 18:670-676.

Hung KY, Huang JW, Chiang CK, and Tsai TJ. 2008. Preservation of peritoneal morphology and function by pentoxifylline in a rat model of peritoneal dialysis: molecular studies. Nephrology Dialysis Transplantation 23:3831-3840. 10.1093/ndt/gfn369

Jafari-Sabet M, Shishegar A, Saeedi AR, and Ghahari S. 2015. Pentoxifylline Increases Antiadhesion Effect of Streptokinase on Postoperative Adhesion Formation: Involvement of Fibrinolytic Pathway. Indian J Surg 77:837-842. 10.1007/s12262-013-1025-y

Kucich U, Rosenbloom JC, Shen G, Abrams WR, Hamilton AD, Sebti SM, and Rosenbloom J. 2000. TGF-betal stimulation of fibronectin transcription in cultured human lung fibroblasts requires active geranylgeranyl transferase I, phosphatidylcholine-specific phospholipase C, protein kinase C-delta, and p38, but not erk1/erk2. Arch Biochem Biophys 374:313-324. 10.1006/abbi.1999.1625

Lamouille S, Xu J, and Derynck R. 2014. Molecular mechanisms of epithelial-mesenchymal transition. Nature Reviews Molecular Cell Biology 15:178-196. 10.1038/nrm3758

Lauder CIW, Garcea G, Strickland A, and Maddern GJ. 2011. Use of a Modified ChitosanDextran Gel to Prevent Peritoneal Adhesions in a Rat Model. Journal of Surgical Research 171:877-882. 10.1016/j.jss.2010.06.028

Lee MTG, Lee CC, Wang HM, Chou TH, Wu MC, Hsueh KL, and Chen SC. 2016. Hypothermia Increases Tissue Plasminogen Activator Expression and Decreases Post-Operative IntraAbdominal Adhesion. Plos One 11. ARTN e0160627

10.1371/journal.pone.0160627

Liakakos T, Thomakos N, Fine PM, Dervenis C, and Young RL. 2001. Peritoneal adhesions: Etiology, pathophysiology, and clinical significance - Recent advances in prevention and management. Digestive Surgery 18:260-273. Doi 10.1159/000050149

Luijendijk RW, deLange DCD, Wauters CCAP, Hop WCJ, Duron JJ, Pailer JL, Camprodon BR, Holmdahl L, vanGeldorp HJ, and Jeekel J. 1996. Foreign material in postoperative adhesions. Annals of Surgery 223:242-248. Doi 10.1097/00000658-199603000-00003

Margetts PJ, Bonniaud P, Liu LM, Hoff CM, Holmes CJ, West-Mays JA, and Kelly MM. 2005. Transient overexpression of TGF-beta 1 induces epithelial mesenchymal transition in the rodent peritoneum. Journal of the American Society of Nephrology 16:425-436. Doi 10.1681/Asn.2004060436

McCarty MF, O'Keefe JH, and DiNicolantonio JJ. 2016. Pentoxifylline for vascular health: a brief review of the literature. Open Heart 3:e000365. 10.1136/openhrt-2015-000365

Menzies D, and Ellis H. 1990. Intestinal-Obstruction from Adhesions - How Big Is the Problem. 
442

443

444

445

446

447

448

449

450

451

452

453

454

455

456

457

458

459

460

461

462

463

464

465

466

467

468

469

470

471

472

473

474

475

476

477

Annals of the Royal College of Surgeons of England 72:60-63.

Oncel M, Kurt N, Remzi FH, Sensu SS, Vural S, Gezen CF, Cincin TG, and Olcay E. 2001. The effectiveness of systemic antibiotics in preventing postoperative, intraabdominal adhesions in an animal model. Journal of Surgical Research 101:52-55. 10.1006/jsre.2001.6245

Otani S, Kuinose M, Murakami T, Saito S, Iwagaki H, Tanaka N, and Tanemoto K. 2008. Preoperative oral administration of pentoxifylline ameliorates respiratory index after cardiopulmonary bypass through decreased production of IL-6. Acta Medica Okayama 62:69-74.

Parra-Membrives P, Ruiz-Luque V, Escudero-Severin C, Aguilar-Luque J, and Mendez-Garcia V. 2007. Effect of pentoxifylline on the healing of ischemic colorectal anastomoses. Diseases of the Colon \& Rectum 50:369-375. 10.1007/s10350-006-0803-z

Piera-Velazquez S, Li ZD, and Jimenez SA. 2011. Role of Endothelial-Mesenchymal Transition (EndoMT) in the Pathogenesis of Fibrotic Disorders. American Journal of Pathology 179:1074-1080. 10.1016/j.ajpath.2011.06.001

Pollice PE, Rosier RN, Looney RJ, Puzas JE, Schwarz EM, and O'Keefe RJ. 2001. Oral pentoxifylline inhibits release of tumor necrosis factor-alpha from human peripheral blood monocytes - A potential treatment for aseptic loosening of total joint components. Journal of Bone and Joint Surgery-American Volume 83a:1057-1061.

Rossner M, and Muller R. 1987. On the Assessment of the Efficacy of Pentoxifylline (Trental). Journal of Medicine 18:1-15.

Saltzman AK, Olson TA, Mohanraj D, Carson LF, and Ramakrishnan S. 1996. Prevention of postoperative adhesions by an antibody to vascular permeability factor/vascular endothelial growth factor in a murine model. American Journal of Obstetrics and Gynecology 174:1502-1506. Doi 10.1016/S0002-9378(96)70596-3

Sulaiman H, Dawson L, Laurent GJ, Bellingan GJ, and Herrick SE. 2002. Role of plasminogen activators in peritoneal adhesion formation. Biochemical Society Transactions 30:126131.

Tarhan OR, Barut I, Sutcu R, Akdeniz Y, and Akturk O. 2006. Pentoxifylline, a methyl xanthine derivative, reduces peritoneal adhesions and increases peritioneal fibrinolysis in rats. Tohoku Journal of Experimental Medicine 209:249-255. DOI 10.1620/tjem.209.249

ten Broek RPG, Issa Y, van Santbrink EJP, Bouvy ND, Kruitwagen RFPM, Jeekel J, Bakkum EA, Rovers MM, and van Goor H. 2013. Burden of adhesions in abdominal and pelvic surgery: systematic review and met-analysis. Bmj-British Medical Journal 347. ARTN f5588

10.1136/bmj.f5588 
478 Vlahos NE, Gregoriou O, Deliveliotou A, Perrea D, Vlachos A, Zhao YL, Lai J, and Creatsas G. 479 2010. Effect of pentoxifylline on vascular endothelial growth factor $\mathrm{C}$ and flk-1 480 expression on endometrial implants in the rat endometriosis model. Fertility and Sterility $48193: 1316-1323.10 .1016 /$ j.fertnstert.2008.10.056

482 Wen WX, Lee SY, Siang R, and Koh RY. 2017. Repurposing Pentoxifylline for the Treatment of 483 Fibrosis: An Overview. Adv Ther 34:1245-1269. 10.1007/s12325-017-0547-2

484 


\section{Table 1 (on next page)}

Scoring system for intra-abdominal adhesion. 


\begin{tabular}{ll}
\hline Score & Adhesion grading scale \\
\hline \hline 0 & No adhesion \\
1 & Thin filmy adhesion \\
2 & More than one thin adhesion \\
3 & Thick adhesion with focal point \\
4 & Thick adhesion with planar attachment \\
5 & Very thick vascular adhesion or more than one planar adhesion \\
\hline
\end{tabular}


Figure 1

Intra-abdominal adhesion score.

The PA+PTX group had lower adhesion score. Data are expressed as the median $\pm I Q R .{ }^{*} P<$ $0.05,{ }^{* *} P<0.01,{ }^{* * *} P<0.001$, respectively.

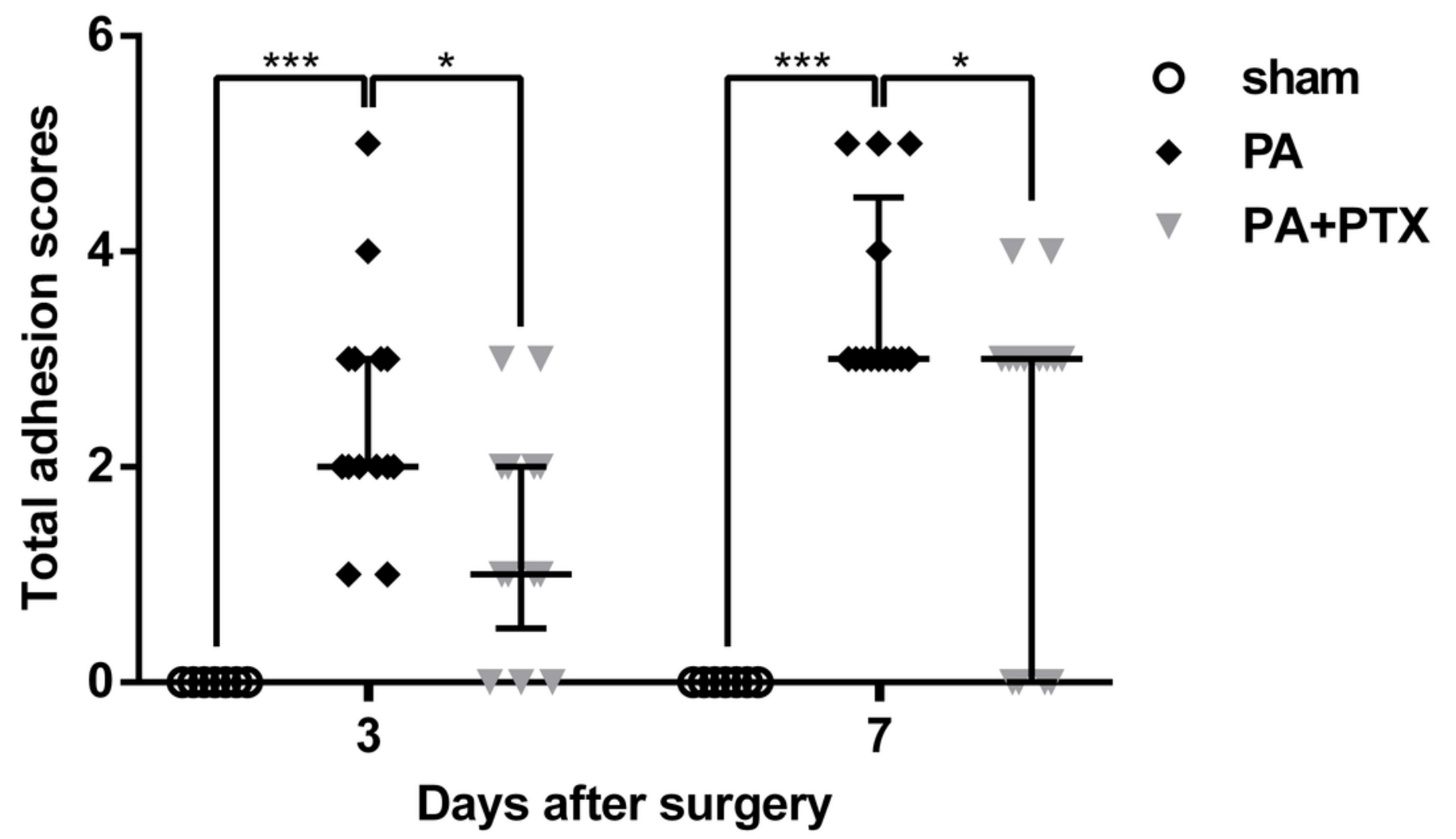


Figure 2

Pentoxifylline Treatment Inhibits Collagen Deposition

(A) Representative images of HE staining. No adhesion was observed in the sham group.

Severe Liver or bowel adhesion and was observed in the PA group, whereas PTX treated

group decreased adhesion severity. (B) Representative images of picrosirius red staining. The thickness of the collagen deposition was increased in PA group, whereas PTX treated group has less collagen deposition. (Original magnification, $x 200$, bar $=100 \mu \mathrm{m}$ ).

A

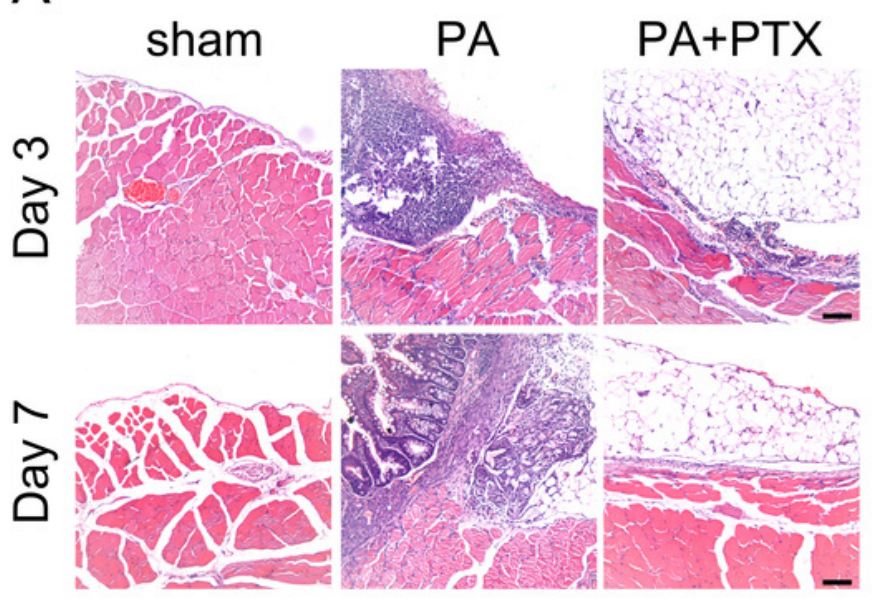

B

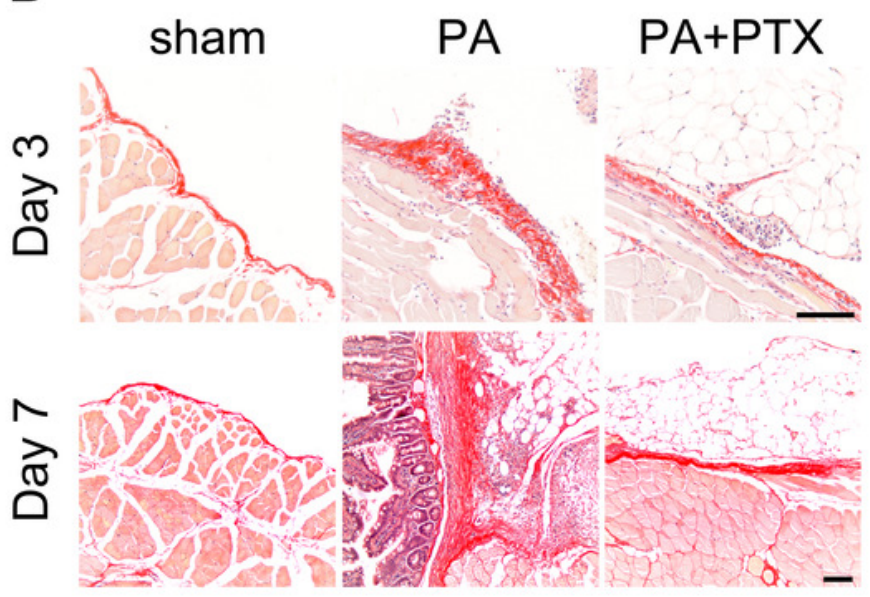




\section{Figure 3}

The levels of tPA protein in peritoneum .

tPA protein level increased in both PA group and PA+PTX group on postoperative day 3 and day7. PTX-treated mice increased tPA protein level at 7 day postoperative compared with 3 day postoperative. Data are expressed as the mean \pm SE. ${ }^{*} P<0.05,{ }^{* *} P<0.01,{ }^{* * *} P<0.001$, respectively.

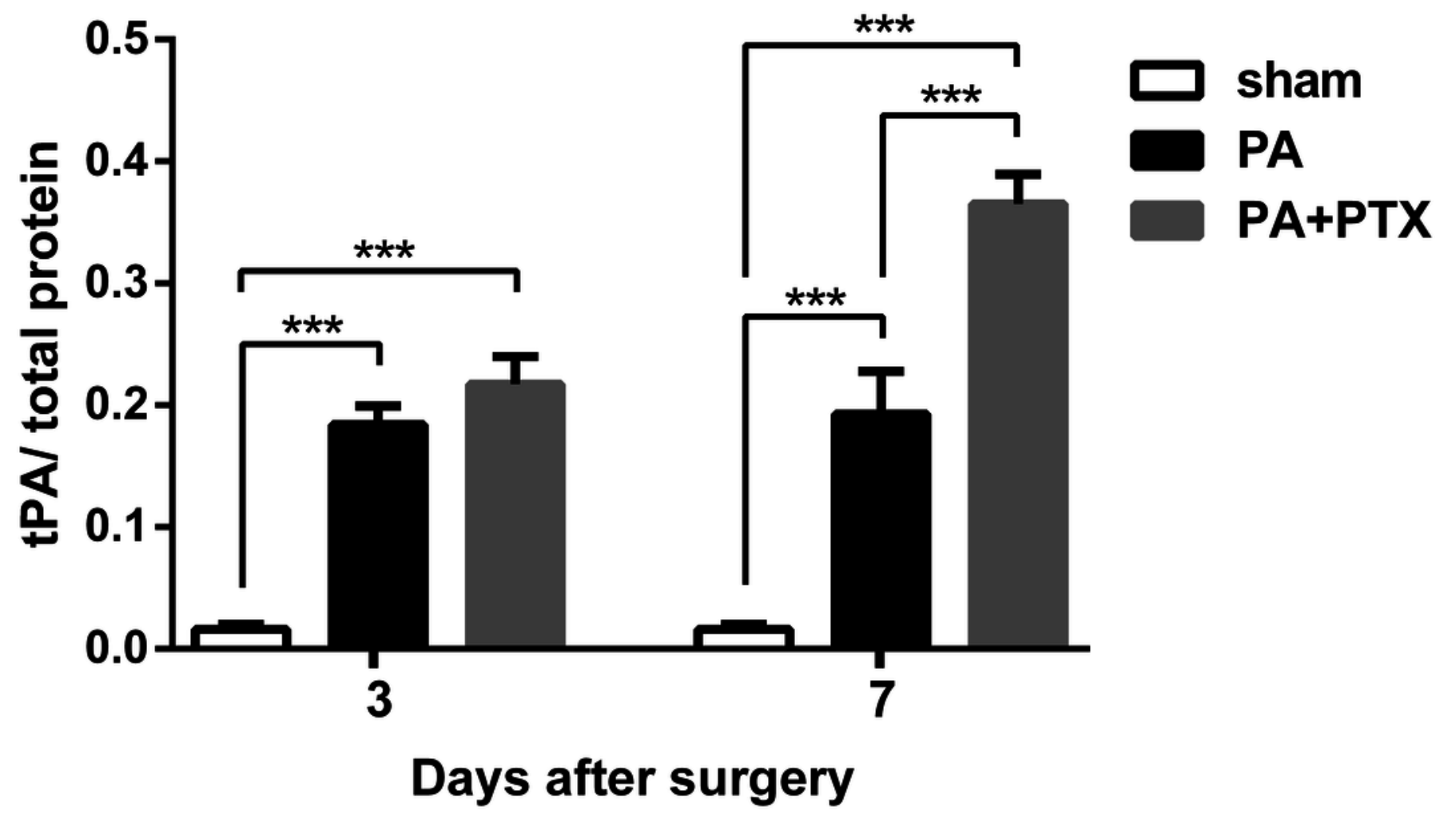




\section{Figure 4}

Pentoxifylline Treatment Reduced Angiogenesis.

(A) Representative examples of double immunohistochemistry staining of ki67 (blue) and CD31 (brown-red) (arrows) in peritoneum on day 3 and day 7. (Original magnification, $x 200$, bar $=100 \mu \mathrm{m})$. (B) The graph shows numbers of cells expressing $\mathrm{Ki}^{+} 7^{+}$and $\mathrm{CD} 31^{+}$ (proliferating endothelial cells) on day 3 and day 7. (C) The graph shows the percentage of $\mathrm{CD} 31^{+}$vessel area per field at $\times 200$ magnification on day 3 and day 7 . Data are expressed as the mean \pm SE. ${ }^{*} P<0.05,{ }^{* *} P<0.01,{ }^{* * *} P<0.001$, respectively.

A
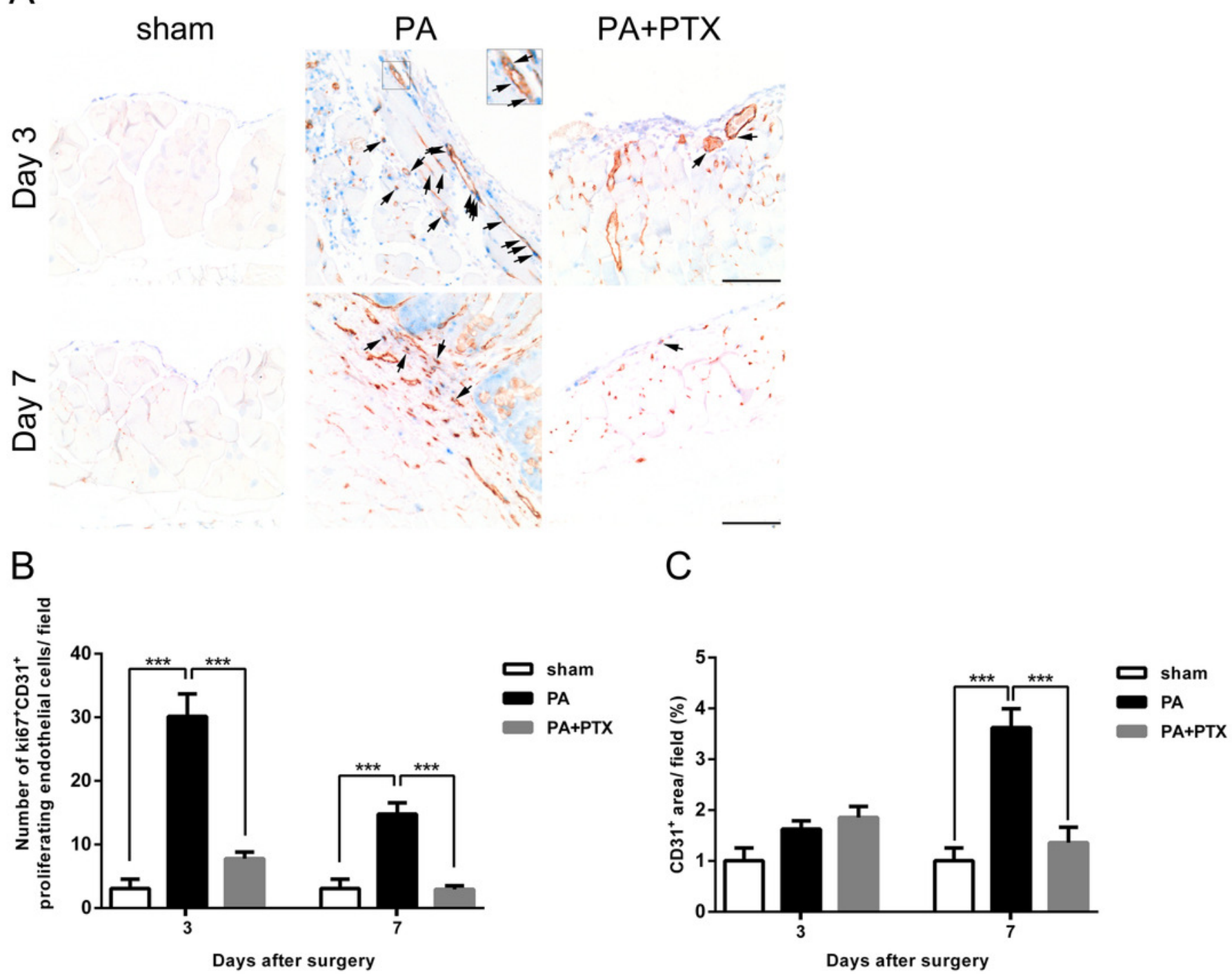


\section{Figure 5}

Pentoxifylline Treatment Reduced Inflammation

(A) Immunohistochemistry for F4/80 was performed on mice peritoneal tissue in the different groups at day 3 and day 7. The F4/80 expression was increased in PA group. Representative images of the sham group, PA group, and PA+PTX group are shown (Original magnification, $\mathrm{x} 200$, bar $=100 \mu \mathrm{m}) .(\mathrm{B})$ Quantification of F4/80+ cells (\%) in high-powered field (HPF) at x400 magnification. Data are expressed as the mean \pm SE. ${ }^{*} P<0.05,{ }^{* *} P<0.01,{ }^{* * *} P<0.001$, respectively.

A

sham

PA

$\mathrm{PA}+\mathrm{PTX}$

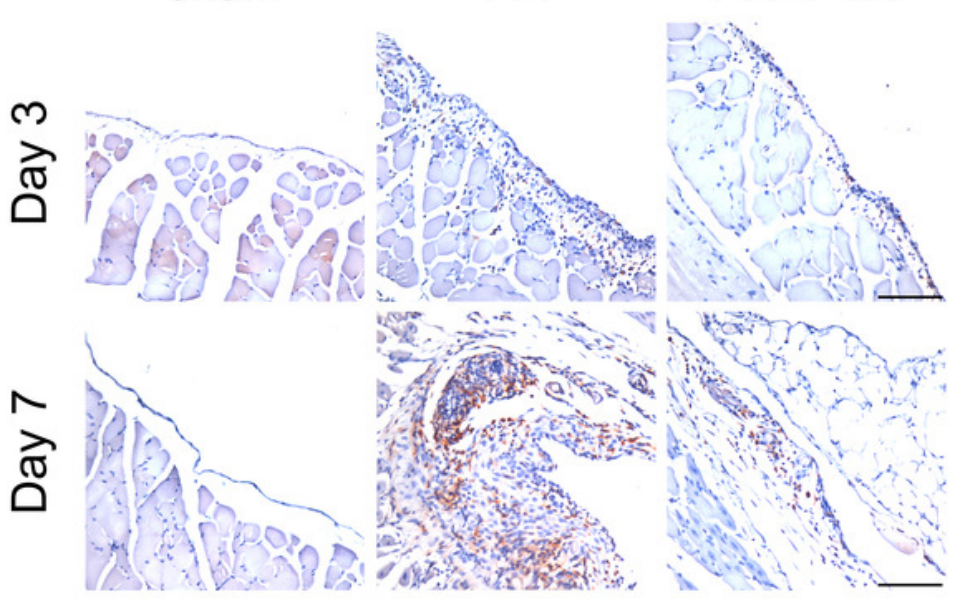

B

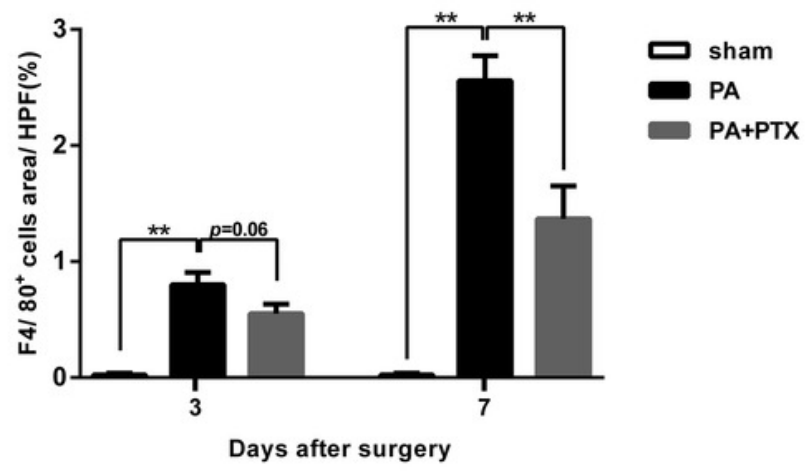




\section{Figure 6}

Pentoxifylline Treatment Reduced the Expression of Fibrosis Marker FSP1

(A-B) Immunohistochemistry for FSP-1 was performed on mice peritoneal tissue in the different groups at day 3 and day 7. The FSP-1 expression was increased in PA group. Representative images of the sham group, PA group, and PA+PTX group are shown. (Original magnification, $x 100$, bar $=100 \mu \mathrm{m})$. (C) Quantification of FSP-1 ${ }^{+}$cells $(\%)$ in high-powered field (HPF) at x400 magnification. Data are expressed as the mean $\pm \mathrm{SE}$. ${ }^{*} P<0.05,{ }^{* *} P<$ $0.01,{ }^{* * *} P<0.001$, respectively.

A

\section{3 day after surgery}

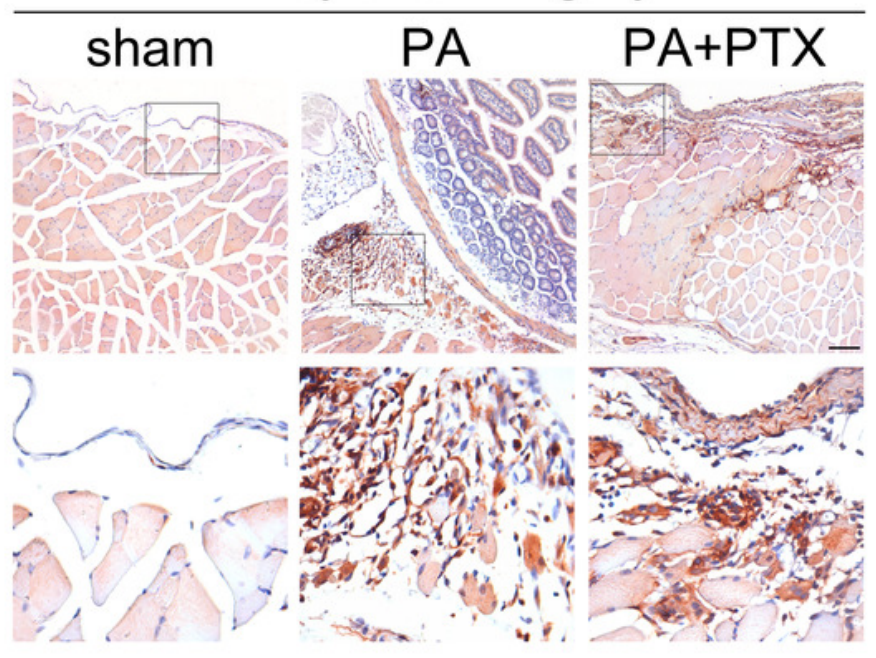

C

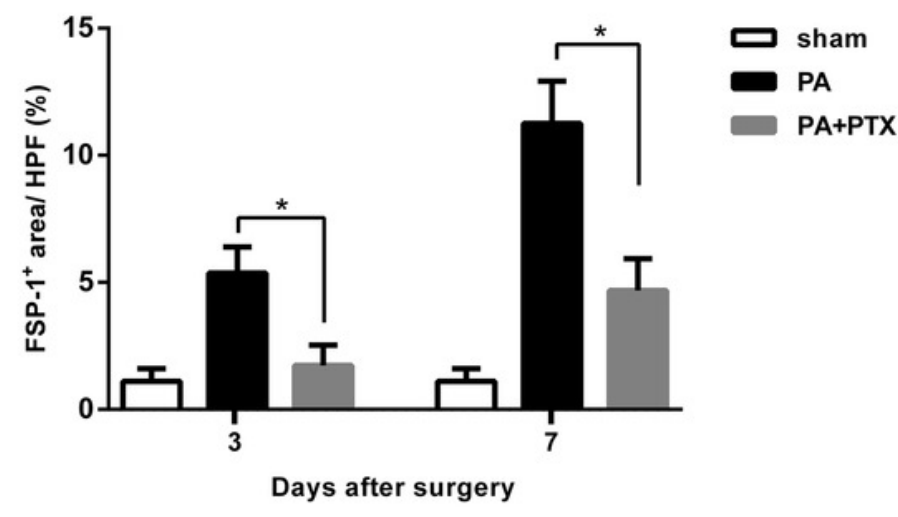

B

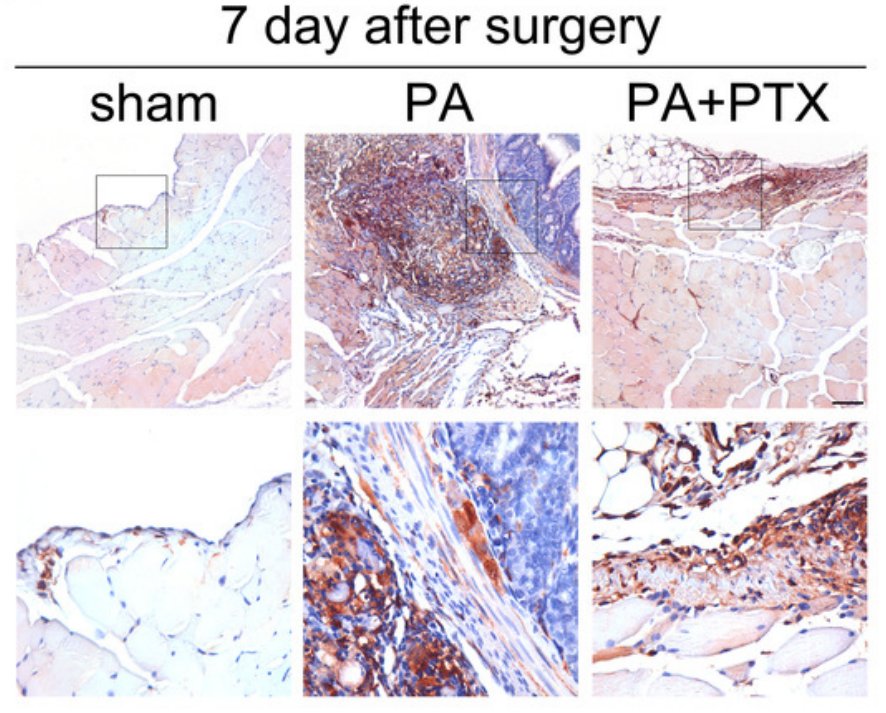




\section{Figure 7}

Pentoxifylline Treatment Reduced the Expression of Fibrosis Marker $\alpha$-SMA

(A-B) Double immunofluorescence was performed with CK18 and $\alpha$-SMA in mice peritoneum in the different groups at day 3 and day 7. Immunofluorescence shows the staining of mesothelial cells by CK18 was express ed in green color, and myofibroblast by $\alpha$-SMA was expressed in red color. In PA group, we observed a few $\mathrm{CK} 18^{+}$cells was co-localization with $\alpha$-SMA in the mesothelial layer. (Original magnification, $x 400$, bar $=100 \mu \mathrm{m}$ ). (C) Quantification of $\alpha$-SMA ${ }^{+}$cells (\%) in high-powered field (HPF) at $\times 400$ magnification. Data are expressed as the mean \pm SE. ${ }^{*} P<0.05,{ }^{* *} P<0.01,{ }^{* * *} P<0.001$, respectively. 
A

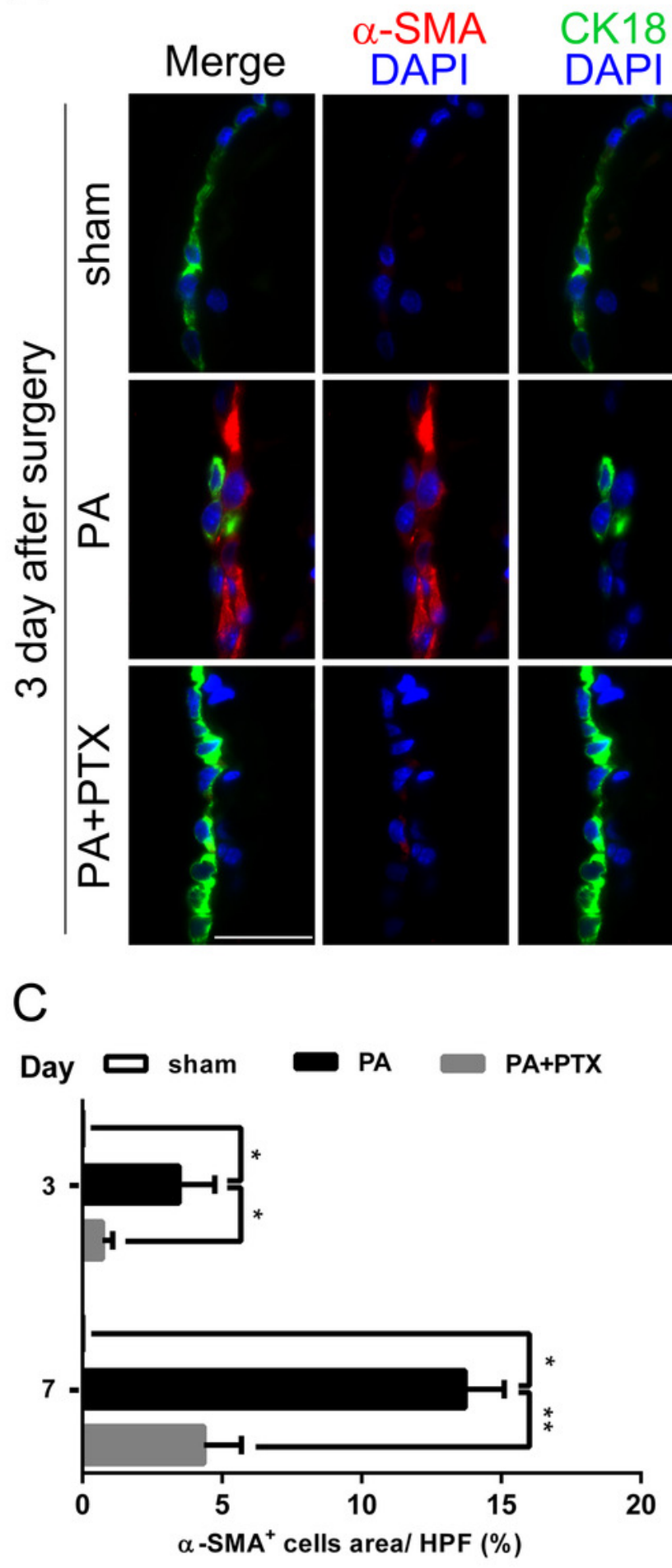

B

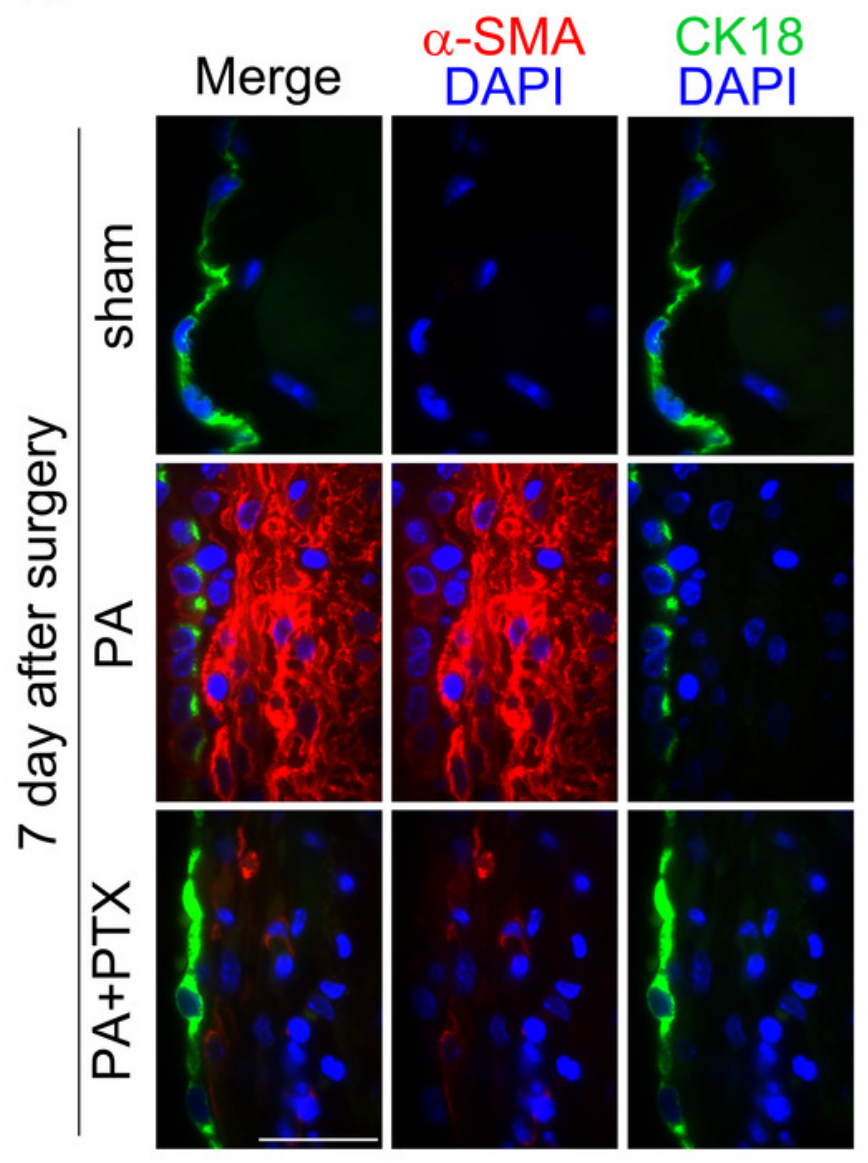

